\title{
Analysis of Various Algorithms and Provide a Graph Theory Approach towards Speeding of Image Reconstruction with Reduced Iterations
}

\author{
V. Dhineshkumar, S. Gobhinath, and S. Vimalraj
}

\begin{abstract}
- this paper makes a comparison between the slow globally convergent list-mode COSEM and the fast but nonconvergent classical list-mode OSEM methods and produce a way to get a fast and effective COSEM called the enhanced COSEM (ECOSEM). The former globally convergent algorithm is based on the ordered sets expectation maximization algorithm for binned data but has several extensions that make it suitable for two planar detector tomography that are rotating but experience certain limitations. The image estimate measured from the PET scanner is incrementally updated for each coincidence event measured. The events obtained are used as soon as possible, that improves the current image estimate, and therefore the convergence speed towards the maximum-likelihood is accelerated. We provide a simulated result to overcome those limitations and to reconstruct the image with maximumlikelihood.
\end{abstract}

Index Terms-Maximum-likelihood (ML), positron emission tomography (PET), row-action maximum-likelihood algorithm (RAMLA), expectation-maximization (EM), event-by-event (EBE), ordered subsets (OS), Bayesian, filtered back projection (FBP), maximum a-posteriori (MAP), penalized weighted least-squares (PWLS).

\section{INTRODUCTION}

Statistical reconstruction of medical images has become more and more popular in emission computed tomography due to its accuracy in modeling the noise, in imaging physics, and incorporates the prior knowledge about the object. There are challenges associated with the practical reconstruction of vast noisy low count data in emission tomography. Positron emission tomography (PET) is widely used in the acquisition of images in the medical field [4]. Based on the measured data it is necessary to evaluate the parameters that are required for the image reconstruction. The internal structure's reconstruction is carried out for the objects that diffuse the radiation. The kinds of radiations include the photons, phonons or neutrons. Actual practice, reconstruction of images from PET is done with the estimation of parameters that are unknown from data obtained through the detection of radiation. There are many

Manuscript received on March 18, 2011; revised July 26, 2011.

S. Vimalraj is the lecturer of EEE Department, VLB Janakiammal College of Engineering and Technology, Coimbatore, India. E-mail: vimalrajeee@gmail.com

V. Dhineshkumar is currently doing his Bachelor's degree in Electrical and Electronics Engineering, VLB Janakiammal College of Engineering and Technology, Coimbatore, India. E-mail: dhinesh89@gmail.com

S. Gobhinath is currently doing his Bachelor's degree in Electrical and Electronics Engineering, VLB Janakiammal College of Engineering and Technology, Coimbatore, India. E-mail: s.gobhinath@gmail.com algorithms available for the estimation of these parameters and also to reconstruct the image obtained.

In One practical limitation associated with the statistical reconstruction method is its slow convergence and this should be improved to have a better reconstructed image. One of the most convenient and widely used algorithms is the FBP (Filtered back projection). But the geometries of the image were not fully acquired by the FBP and its analytical relatives. In addition, the performance shows that the FBP was outperformed by the statistical reconstructions. The maximum-likelihood (ML) algorithm [1] is the one which produces the better quality images, but with the increase in the iteration process the noise properties in the images tend to deteriorate. To avoid this deterioration the Bayesian method was developed [7]. The principle of this method is that it incorporates the priori information of the image to improve the resulting one with good quality. However, with the introduction of improved Bayesian the speed of convergence was a question in this method [8]. The penalized weighted least-squares (PWLS) image reconstruction of PET data is an effective means of estimating the data from an image, but the limitation with this method is the computational problem [12]. The formulae involving in this method of reconstruction is quite complex and also takes a bit long for the computation. So there is a need for the algorithm with fast and convergent in the case of statistical image reconstruction.

There are five important components required for the statistical image reconstruction:

1) There must be positron-annihilation distribution with finite parameterization, e.g., representation of a discretized image,

2) The model should relate the image to the expected measurement of each detector which is unknown with high level of accuracy,

3) The variations in the detectors measurement around the expectation should be available in the statistical model,

4) The estimation of image is found with the maximization of an objective function, and

5) There should be an algorithm which is typically an iterative one which maximizes the objective function and includes the specification of stopping criteria and initial estimate.

Later two widely used approaches maximum-likelihood (ML) and maximum a-posteriori (MAP) were developed which are performing on the basis of iterative algorithms. The former possess the algorithms like expectation maximization (EM), row-action maximum-likelihood algorithm (RAMLA), etc. and the later includes the 
algorithms like BSREM, relaxed OS-PS, etc [13]. The MAP has some limitations such as the increase in iterations in the order of 104-105 and also there is no control over the noise elimination process while handling large sets of data. The ML algorithm forms an effective basis for the iterative reconstruction of medical images with reduced noise level nearly $50 \%$ greater than other algorithms.

The two forms of expectation maximization deals with the handling of data sets. OSEM (Ordered Subset EM) is fast but non-convergent, handles the data of present image estimate alone, producing reconstructed image with minimum number of iterations. COSEM (Complete data OSEM) is the globally convergent algorithm which handles both the present and past estimates of images. It is an incremental EM algorithm with COSEM-ML for maximumlikelihood and COSEM-MAP for maximum a-posteriori [6] Moreover it does not require the object dependent userspecified relaxation schedule. The suited iterative approach for the reconstruction is COSEM-ML which in turn involves list mode (LM) and histogram. List mode allows the collection of data and its attributes at higher precision levels than the binned one (histogram). The LM suffers from the increased utilization of memory for the storage of current and previous estimate and incrementally updating the event counts while handing more number of events $(L>1)$ per subset [11]. To minimize the utilization of memory the number of events per subset was reduced to its minimum level as one thus forming the new process work event-byevent (EBE).

\section{COSEM-MAP}

The MAP (Maximum a-posteriori) produces a good reconstruction of the emission tomography. The measurements associated with the Positron Emission Tomography, $y_{j}, j=1, \ldots \ldots, M$ are modeled to be as an independent Poisson random variables, that is,

$$
y_{j} \sim \operatorname{Poisson}\left(\sum_{i=1}^{N} \lambda_{i} p_{i j}\right) \text { for } j=1, \ldots . ., M,
$$

where $\lambda_{i}, i=1, \ldots \ldots, N$.

The above equations are considered to the mean of the emission process. The annihilation of the $i^{\text {th }}$ pixel is detected with the help of $j^{\text {th }}$ detector with a probability of $p_{i j}$. The individual Poisson processes joint probability $Y=y$ is with the observation of condition probability i.e. likelihood function $\Lambda=\lambda$. The density function $P(\lambda / y)$ of posterior is maximized with the estimate $\lambda^{M L}$ determined by the MAP. With the suitable prior $P(\lambda)$ the reconstruction of MAP is formulated as,

$$
\lambda^{M A P}=\max _{\lambda \geq 0}[\log P(y / \lambda)+\log P(\lambda)]
$$

The Gibbs distribution is used for characterization of the image $\lambda$ with the assumption of image field to be Markov random field.

$$
P(\lambda)=\frac{1}{Z} \exp \left\{-\frac{1}{\beta} U(\lambda)\right\}
$$

where, $Z$ is characterized as normalizing constant and $\beta$ as the Gibbs hyper-parameter. The Gibbs energy is given to be $U(\lambda)=\sum_{i} \sum_{j \in N_{i}} \omega_{i j} V\left(\lambda_{i}, \lambda_{j}\right)$. The $\omega_{i j}$ is given as the weight of the pixel $j \in N_{i}$, with $N_{i}$ as the nearest neighbor set pixel of $i$. The nearest neighbor element $j \in N_{i}$ provides some potential $V\left(\lambda_{i}, \lambda_{j}\right)$ at site $i$. The equation (1) is complex due to the nature of prior.

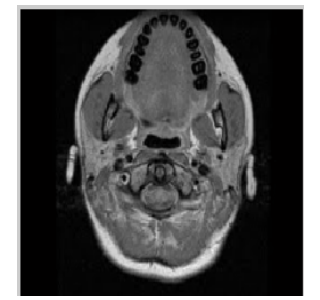

(a)

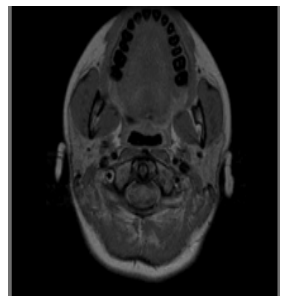

(b)
Fig.1. (a) Original PET phantom. (b) Reconstructed phantom using MAP

Impose of positivity in the equation with the inclusion of Lagrange series is not necessarily needed because the MAP already includes the series within it. But however with the increase in iterations, processing of noise is not effective and also suffers from over smoothening effect. Fig. 1 shows the original phantom image and the reconstructed image using the MAP algorithm [14]. It is clearly seen that the reconstructed image is suffering from noise and is over smoothed.

\section{LIST-MODE COSEM}

The estimation of image is noted to be $\mathrm{M}$ for the bin data containing $\mathrm{N}$ events in all statistical list-mode algorithms. The complete data of the list-mode is partitioned into a number of subsets L [5].

$$
N=\stackrel{L-1}{\uplus_{l=0}} S_{l}
$$

With $0 \leq l<L$, and $1 \leq L \leq|N|$. The subset $S_{l} \subset N$ contains nearly equal number of events. Where the 0 represents gray scale level black and L-1 represents the gray scale level white. The image to be reconstructed is enhanced in prior to reduce the noise handling process.

Successive bin values of each image are related in the case of iterative image reconstruction. The intermediate formula for this iterative algorithm is,

$$
\hat{\lambda}_{j}^{n+1}=\frac{\lambda_{j}^{n}}{\omega_{j}} \sum_{i \in S_{l}} \frac{A_{i j}}{a_{i} \sum_{k \in M} A_{i k} \lambda_{k}{ }^{n}}, l=\left[\frac{n \bmod |N|}{L}\right]
$$

Equation (5) is defined as the basic building block for the construction of list-mode algorithm. LM-COSEM [2] is developed from the LM-OSEM which is the fast but non convergent algorithm. The LM-OSEM replaces the current estimate of the image with the next subset values and uses more than one event $(\mathrm{L}>1)$. With the intermediate result from (5) we obtain the result to be,

$$
\lambda_{j}{ }^{n+1}=L \hat{\lambda}_{j}{ }^{n+1}
$$

The maximum likelihood approach should be provided with a minimized objective function and is given by,

$$
\hat{f}=\arg \min _{f \geq 0} E_{\text {inc }}-{ }_{M L}(f)
$$

The Poisson likelihood [3] associated with the ML algorithm is given as, 


$$
\begin{aligned}
E_{\text {inc }}-{ }_{M L}(f) & =-\log \operatorname{Pr}(g \mid f) \\
& =\sum_{i j} H_{i j} f_{j}-\sum_{i} g_{i} \log \sum_{j} H_{i j} f_{j}
\end{aligned}
$$

LM-OSEM takes only a subset $S_{l} \subset N$ of data to make an evaluation of multiplicative correction factor for each separate bin. Each event is processed and the images are updated for each and every sub-iteration levels. The process continues until all the available events are estimated. Whereas, the LM-COSEM sums up the intermediate results to form the globally convergent results and can be denoted as,

$$
\lambda_{j}^{n+1}=\sum_{s=0}^{L-1} \hat{\lambda}_{j}^{n+1-s}
$$

In LM-COSEM the incremental update is used as a new term which sums up to replace the previous image contributions. The corresponding incremental update is equivalent to,

$$
\lambda_{j}{ }^{n+1}=\lambda_{j}{ }^{n}+\hat{\lambda}_{j}^{n+1}-\hat{\lambda}_{j}^{n+1-L}
$$

As discussed earlier the COSEM algorithm suffers from slow convergent process. To rectify this, a special combination of OSEM and COSEM was developed and so called the Enhanced COSEM (ECOSEM). This method can be obtained from the combination of (6) and (10).

$$
\lambda_{j}{ }^{n+1}=t(n) L \hat{\lambda}_{j}^{n+1}+(1-t(n))\left(\lambda_{j}{ }^{n}+\hat{\lambda}_{j}^{n+1}-\hat{\lambda}_{j}^{n+1-L}\right)(11)
$$

ECOSEM works on the principle that, the OSEM which is the fast but non convergent algorithm with which the image reconstruction is started, then the globally convergent COSEM is implemented on to the images to produce a reconstructed image. Thus the algorithm becomes faster and also convergent. The speed and convergence are balanced by the trade-off parameter $t(n)$ with $0 \leq t(n) \leq 1$. The practical implementation of list-mode COSEM produces a result far better than the MAP-COSEM and also the resultant formulae available for the COSEM is not much complex in comparison with the other methods of iterative reconstruction [15]. The phantom image obtained from the PET scanner is used in this LM-COSEM and the reconstructed image is obtained.

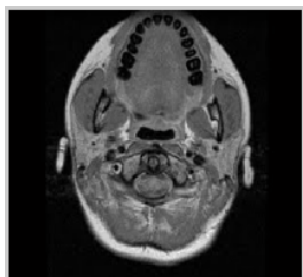

(a)

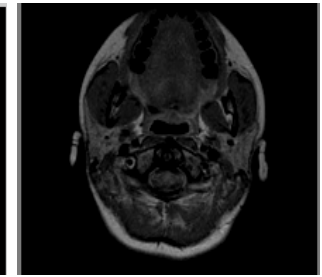

(b)
Fig.2. (a) Original PET phantom. (b) Reconstructed phantom using LMCOSEM.

\section{Event-By-Event Cosem}

One of the particular cases of LM-COSEM is the EBECOSEM in which the number of events associated with each subset is limited to one i.e. as small as possible and can be characterized as $L=N$. With subset containing only one event we have, $S_{i}=\{i\}$. With these data we have the intermediate equation (5) be,

$$
\hat{\lambda}_{j}^{n+1}=\frac{A_{i j} \lambda_{j}{ }^{n}}{\omega_{j} a_{i} \sum_{k \in M} A_{i j} \lambda_{k}{ }^{n}}, i=n \bmod |N|
$$

Thus we obtain with the intermediate equation. But the actual EBE-COSEM algorithm's equation can be obtained by inserting (10) in (8) we have,

$$
\lambda_{j}^{n+1}=\lambda_{j}^{n}+\frac{A_{i j}}{\omega_{j} a_{i}}\left[\frac{\lambda_{j}^{n}}{\sum_{k \in M} A_{i j} \lambda_{k}{ }^{n}}-\frac{\lambda_{j}^{n-|N|}}{\sum_{k \in M} A_{i j} \lambda_{k}^{n-|N|}}\right]
$$

where $i=n \bmod |N|$.

Working with the singleton subset in LM-COSEM, the image bin $j \in M$ of each event $i \in N$ is updated at each step. In the very first iteration, the negative term in the equation (13) is equal to zero and so $n-|N|<0$, then $\lambda_{j}^{n-|N|}=0$ if $0 \leq n<N$. Therefore it is not necessary to include the first pass and thus the algorithm can be simplified.

The reconstruction property of event-by-event and its implementation is more feasible because of the locality property in which only the image bin along the Line of Response (LOR) is only modified [9]. It is also to be noted that the computational time is similar to the conventional list-mode COSEM. Incremental update of the image is essential in this case and is given as,

$$
\lambda_{j}{ }^{n+1}=\lambda_{j}{ }^{n}+\frac{A_{i j} \lambda_{j}{ }^{n}}{\omega_{j} a_{i} \sum_{k \in M} A_{i k} \lambda_{k}{ }^{n}}-\Delta_{j}{ }^{n}, i=n \bmod |N|
$$

with

$$
\Delta_{j}{ }^{n}=\left\{\begin{array}{cc}
\lambda_{j}{ }^{n+1-S_{l}}, & \text { if } n+1 \bmod |N| \notin S_{l} \\
0, & \text { otherwise }
\end{array}\right.
$$

where $l=[n \bmod |N| / L]$.

With the consideration to memory constrains EBECOSEM only takes care of the data associated with the present subset while deleting the data of previous subsets.

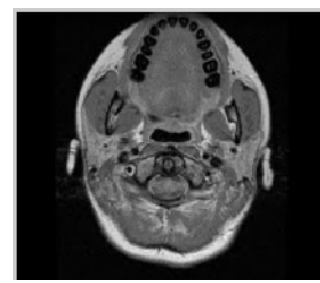

(a)

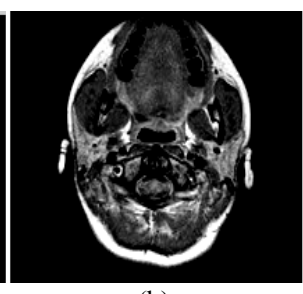

(b)
Fig.3. (a) Original PET phantom. (b) Reconstructed image using EBECOSEM

Among the other iterative reconstructive algorithm the reconstructed image using the EBE-COSEM is more efficient in the case of noise handling and the over smoothening effect. Fig.3 shows the reconstructed image using EBE-COSEM and when compared with the result of Fig.1 and Fig.2, the noise level is reduced and also the smoothening effect is corrected.

\section{Estimation OF PREVIOUS UPDATES}

The calculation of previous image update is ultimately required in the case of EBE-COSEM, but eventually the data were deleted once the next subset calculation is 
initiated. So it is mandatory in re-computing the previous image updates. For the list-mode data, a new online image reconstruction pipeline was formulated with the course of new EBE-COSEM [10]. Two separate routines are dedicated to take care of the first two iterations. Then the arbitrary number of passes of subsets is handled by the recursive algorithms. This type of computation eventually increases the running time of the iterations which became a limitation for using this algorithm.

\section{REDUCTION IN THE COMPUTATION TIME}

Graph theory, an approach to reduce the computational time by reducing the number of steps involved in calculating the iterations, will result in the use of EBECOSEM at ease also reduces the computation time of image reconstruction,

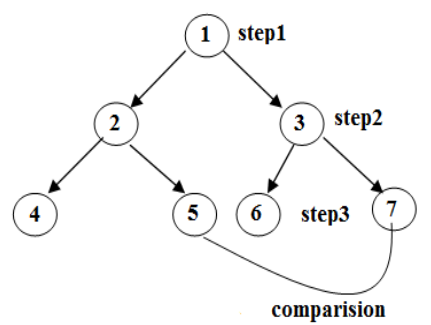

Fig.4. Graph approach

In Fig.4 the numbers 1, 2, 3....7 represents the number of iterations for image reconstruction. Globally convergent list-mode algorithms required 7 iterations are performed simple reconstruction, but in the graph theory approach involving in just 3 steps to obtain 7 iterations. The final output in each leg is then compared to minimize the estimation of statistical elements associated with each event. Then the graph theory approach used to reduce the number of steps involved image reconstruction.

\section{COMAPARITIVE RESUltS}

The convergence effect and the speed of computation between the LM-COSEM and the EBE-COSEM are related to provide a view of making a choice for better reconstruction of medical images.

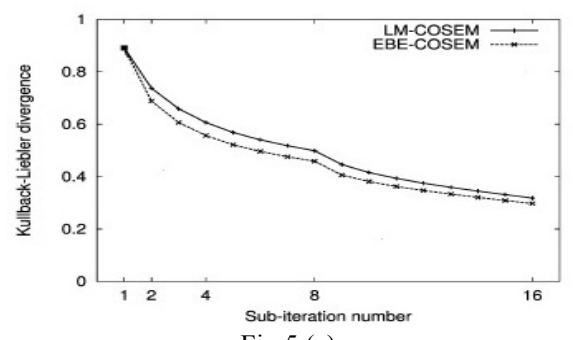

Fig.5 (a)

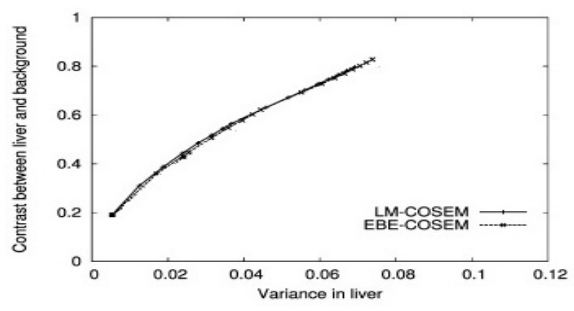

Fig.5 (b)
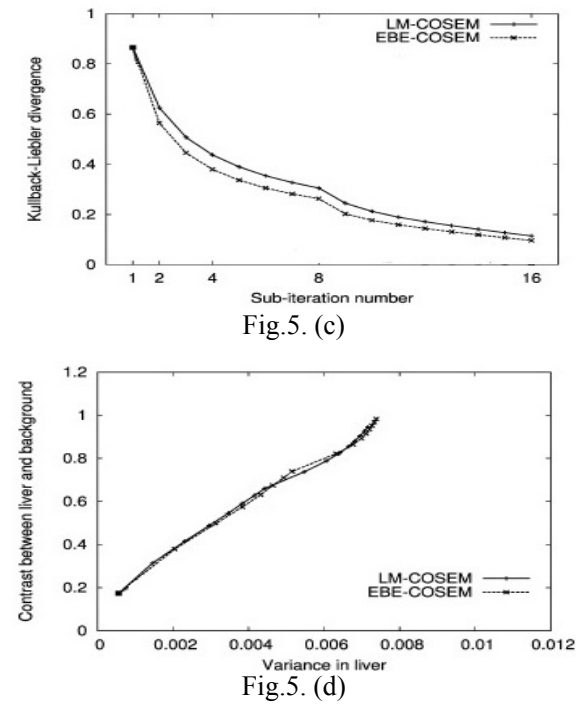

From fig.5 (a) to 5(d), the various simulated graphs for the estimation of statistical elements from the image and their convergence speed is detailed. The liver is taken as an input image that is to be reconstructed. In the above example, for the ease of operations, only 16 iterations were made and their corresponding results are simulated.

\section{CONClusion AND Future Prospectus}

Iterative algorithms involving reconstruction of images should handle the noise process and produce the image with better quality with less number of iterations and less computational time. In this paper we discussed some algorithms like ML, MAP, OSEM, COSEM, LM-COSEM, EBE-COSEM, etc.
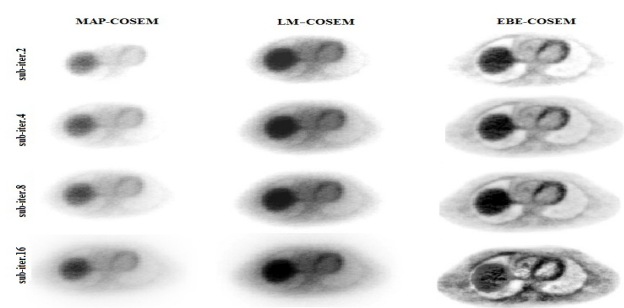

Fig.6. Comparison of outputs obtained from MAP-COSEM, LM-COSEM and EBE-COSEM for the phantom.

The result associated with each case is discussed in detail in analogous with the equations involved in the iterative operations. The convergence speed and memory are the two constraints which play a vital role in selecting the algorithm to be used. An approach to reduce the computational speed was also proposed which drastically reduces the number of steps involved in solving the iterations. In the future work, a process involving the reduction in memory which acts as a main barrier is to be developed. While considering many network processes, the Fuzzy Logic provides a way to reduce the memory which is handled in the iterative process and moreover the iterations involved is also very much reduced. The implementation ECOSEM in fuzzy logic is the next challenge to be encountered.

\section{REFERENCES}

[1] L. A. Shepp and Y. Vardi, "Maximum likelihood reconstruction for emission tomography," IEEE Trans. Med. Imag., vol. MI-4, no. 2, pp. 113-122, Feb. 1982. 
[2] H. Barrett, T. White, and L. Parra, "List-mode likelihood," J. Opt. Soc. Amer. A, vol. 14, no. 11, pp. 2914-2923, 1997.

[3] H. M. Hudson and R. S. Larkin, "Accelerated image reconstruction using ordered subsets of projection data," IEEE Trans. Med. Imag.vol. 13, no. 4, pp. 601-609, Apr. 1994.

[4] A. J. Reader, S. Ally, F. Bakatselos, R. Manavaki, R. J. Walledge, A. P. Jeavons, P. J. Julyan, S. Zhao, D. L. Hasting, and J. Zweit, "Onepass L ist-mode EM algorithm for high resolution 3D PET image reconstruction into large arrays," IEEE Trans. Nucl. Sci., vol. 49, no. 3, pp. 693-699, Mar. 2002.

[5] A. Reader, R. Manavaki, S. Zhao, P. Julyan, D. Hasting, and J. Zweit, “Accelerated list-mode EMalgorithm," IEEE Trans. Nucl. Sci., vol. 49, no. 1, pp. 42-49, Jan. 2002.

[6] A. R. De Pierro, "On the relation between the ISRA and the EM algorithm for positron emission tomography,"IEEE Trans. Med. Imag., vol. 12, no. 2, pp. $328\{333,1993$.

[7] M. Lee, Bayesian Reconstruction In Emission Tomography Using Gibbs Priors, Ph.D. thesis, Yale University, New Haven, CT, 1994.

[8] A. Rangarajan, S.-J. Lee, and G. Gindi, "Mechanical models as priors in Bayesian tomographic reconstruction," in Maximum Entropy and Bayesian Methods, Dordrecht, Netherlands, 1996, pp. 117\{124, Kluwer Academic Publishers\}.

[9] M. E. Phelps, J. C. Mazziotta, and H. R. Schelbert, Positron Emission Tomography and Autoradiography. New York: Raven, 1986.

[10] K. Wienhard, M. Dahlbom, L. Eriksson et al., "The ECAT EXACT HR: Performance of a new high-resolution positron scanner," J. Comput. Assist. Tomogr., vol. 18, pp. 110-118, 1994.

[11] T. R. DeGrado, T. G. Turkington, J. J. Williams et al., "Performance characteristics of a whole-body PET scanner," J. Nucl. Med., vol. 35, pp. 1398-1406, 1994.

[12] H. W. Muller-Gartner, J. M. Links, J. L. Prince et al., "Measurement of radiotracer concentration in brain gray matter using positron emission tomography: MRI-based correction for partial volume effects," J. Cereb. Blood Flow Metab., vol. 12, pp. 571-583, 1992.
[13] Z. Zhou, R. M. Leahy and J. Qi, “Approximate maximum likelihood hyperparameter estimation for Gibbs prior", IEEE Trans. on Img. proc., Vol.6, No.6, pp.844-861, June, 1997

[14] J. Nuyts, D. Bequ, P. Dupont, and L. Mortelmans, "A Concave Prior Penalizing Relative Differences for Maximum-a-Posteriori Reconstruction in Emission Tomography ", IEEE Trans. on Nuclear Science, Vol. 49, No. 1, pp.56-60, Feb. 2002

[15] S. Alenius and U. Ruotsalainen, "Using Local Median as the Location of Prior Distribution in Iterative Emission Tomography Reconstruction”, IEEE Tran. Nucl. Sci., Vol.45, No.6, Dec. 1998.

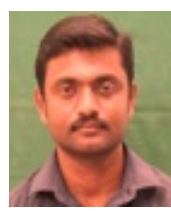

S. Vimalraj received the Bachelors Degree in Electrical and Electronics Engineering in 2006. He completed his Masters degree in Applied Electronics in 2008. He is currently a PhD candidate in the faculty of Electrical and Electronics Engineering, Anna University of Technology, Coimbatore. His current research interests include the testing of VLSI and Embedded Systems.

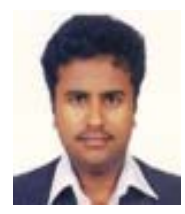

V. Dhineshkumar is currently doing his final year Bachelor's degree in Electrical and Electronics Engineering, VLB Janakiammal College of Engineering and Technology. His areas of interest include the Embedded Systems, Applied Electronics and Micro Electro Mechanical Systems.

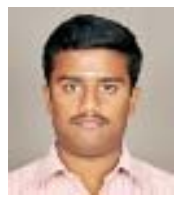

S. Gobhinath born on $5^{\text {th }}$ October 1989 doing his final year Bachelor's degree in Electrical and Electronics Engineering, VLB Janakiammal College of Engineering and Technology. His area of interest includes Automation, Robotics and Embedded systems. 